\title{
Inhibition of apoptosis induced by ischemia-reperfusion prevents inflammation
}

\author{
Marc A.R.C. Daemen, ${ }^{1}$ Cornelis van 't Veer, ${ }^{1}$ Geertrui Denecker, ${ }^{2}$ Vincent H. Heemskerk, ${ }^{1}$ \\ Tim G.A.M. Wolfs, ${ }^{1}$ Matthias Clauss, ${ }^{3}$ Peter Vandenabeele, ${ }^{2}$ and Wim A. Buurman ${ }^{1}$ \\ ${ }^{1}$ Department of General Surgery, University of Maastricht, 6200 MD Maastricht, the Netherlands \\ ${ }^{2}$ Department of Molecular Biology, Flanders Interuniversity Institute for Biotechnology and University of Ghent, \\ B-9000 Ghent, Belgium \\ ${ }^{3}$ Department of Molecular Cell Biology, Max-Planck-Institut für Physiologische und Klinische Forshung, 61231 \\ Bad Nauheim, Germany \\ Address correspondence to: W.A. Buurman, Department of General Surgery, University of Maastricht, PO Box 616, \\ 6200 MD Maastricht, the Netherlands. Phone: 31-4-33-88-14-63; Fax: 31-4-33-88-41-54; E-mail: w.buurman@ah.unimaas.nl.
}

Received for publication April 1, 1999, and accepted in revised form July 13, 1999.

Ischemia followed by reperfusion leads to severe organ injury and dysfunction. Inflammation is considered to be the most important cause of tissue injury in organs subjected to ischemia. The mechanism that triggers inflammation and organ injury after ischemia remains to be elucidated, although different causes have been postulated. We investigated the role of apoptosis in the induction of inflammation and organ damage after renal ischemia. Using a murine model, we demonstrate a relationship between apoptosis and subsequent inflammation. At the time of reperfusion, administration of the antiapoptotic agents IGF-1 and ZVAD-fmk (a caspase inactivator) prevented the early onset of not only renal apoptosis, but also inflammation and tissue injury. Conversely, when the antiapoptotic agents were administered after onset of apoptosis, these protective effects were completely abrogated. The presence of apoptosis was directly correlated with posttranslational processing of the endothelial monocyte-activating polypeptide II (EMAP-II), which may explain apoptosis-induced influx and sequestration of leukocytes in the reperfused kidney. These results strongly suggest that apoptosis is a crucial event that can initiate reperfusion-induced inflammation and subsequent tissue injury. The newly described pathophysiological insights provide important opportunities to effectively prevent clinical manifestations of reperfusion injury in the kidney, and potentially in other organs.

J. Clin. Invest. 104:541-549 (1999).

\section{Introduction}

Central organ systems, including heart, brain, gut, and kidney, are particularly sensitive to ischemia followed by reperfusion (I/R). Renal I/R injury alone imposes a significant burden on the healthcare system because it not only restricts function and availability of donor kidneys for transplantation, but is also a serious complication of circulatory shock.

More specifically, inflammation is considered to be a major cause of I/R-induced tissue injury, because therapeutic strategies aimed at reducing inflammation often ameliorate I/R injury (1). The local inflammatory reaction that follows reperfusion involves cytokines such as proinflammatory TNF- $\alpha$, as well as recruitment of neutrophils. In vitro studies imply certain roles for the anoxia-induced release of chemokines and neutrophil-endothelium interactions $(2,3)$ in the induction of inflammation after I/R. Nevertheless, little is known about the specific events that trigger this inflammatory response in vivo.

In this study, we investigate whether another early consequence of I/R, apoptotic cell death (4), contributes to the inflammatory response in a murine model of renal I/R. Our results challenge the paradigm which states that apoptosis represents a physiological mode of cell deletion allowing disposal of superfluous cells with minimal tissue reaction, or even with active restraining of inflammation (5). We show that exogenous IGF-1 (a growth and survival factor) and the active caspase inhibitor $\mathrm{Z}$-Val-Ala- $\mathrm{Asp}(\mathrm{OMe})-\mathrm{CH}_{2} \mathrm{~F}$ (ZVAD-fmk) attenuate reperfusion-induced inflammation, most likely because of their ability to inhibit apoptosis in vivo. These findings strongly suggest that apoptotic cell death, either directly or indirectly, significantly contributes to I/R-induced inflammation, as well as to the consequent tissue damage, and may provide new therapeutic means to treat I/R injury.

\section{Methods}

Antibodies and reagents. Anti-murine neutrophil $\mathrm{mAb}$ Gr-1 was obtained from PharMingen (San Diego, California, USA); peroxidase-conjugated goat anti-rat and goat anti-rabbit IgGs were obtained from The Jackson Laboratory (Bar Harbor, Maine, USA); peroxidase-conjugated sheep anti-digoxigenin, digoxigenin 11-dUTP, and terminal deoxynucleotidyl transferase (TdT) were obtained from Boehringer Mannheim $\mathrm{GmbH}$ (Mannheim, Germany); peroxidase-conjugated rabbit anti-sheep $\operatorname{IgG}$ was obtained from DAKO A/S (Glostrup, Denmark); the rabbit antiserum SA 2846 

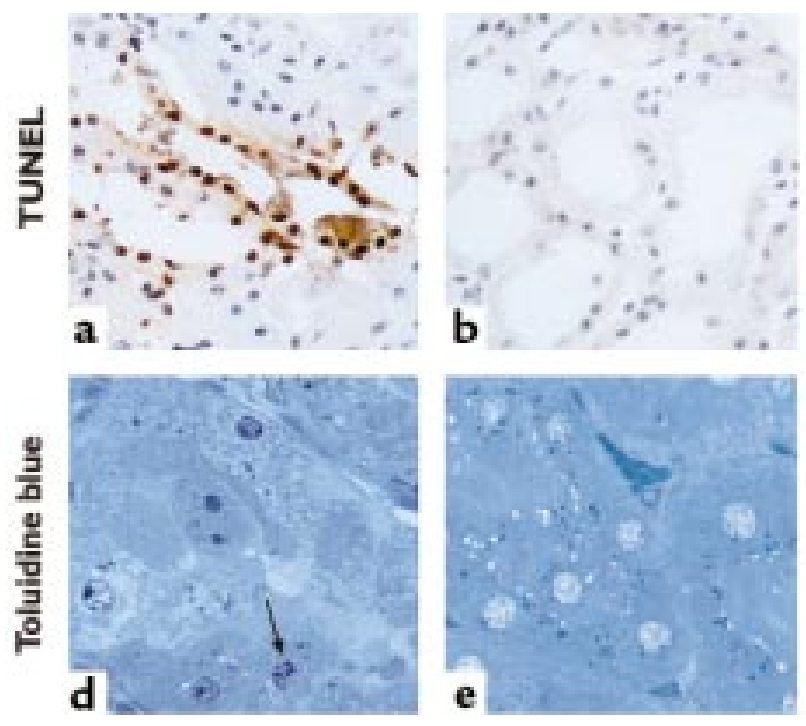

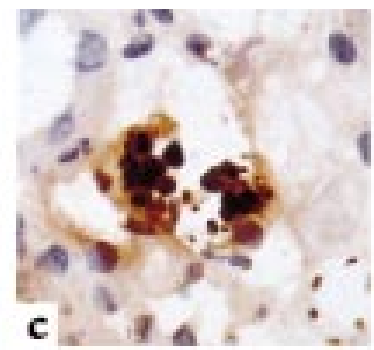

Figure 1

Representative light micrographs showing evident in situ detection of DNA nick ends by TUNEL histology (a-c). The tubular epithelial cells of the outer medulla from biopsies obtained after 2 hours of reperfusion showed positive staining $(\mathbf{a})$, in contrast to kidneys obtained from sham-

operated mice $(\mathbf{b})(\times 250)$. The presence of apoptotic bodies could be detected extracellularly and extruded into the tubular lumen after 2 hours of reperfusion $(\mathbf{c})(\times 1,000)$. Toluidine blue stains of $1-\mu \mathrm{m}$ sections, obtained from kidneys that were fixed in situ after 3 hours of reperfusion and subsequently embedded in plastic ( $\mathbf{d}$ and $\mathbf{e}$ ), revealed the presence of dense condensation of nuclear chromatin and nuclear fragmentation in apoptotic tubular epithelial cells (arrow) in the outer medulla (d), whereas morphological attributes of tubular epithelial cell apoptosis were generally absent in shamoperated mice $(\mathbf{e})(\times 1,000)$.

The animals were sacrificed at different time points, ranging from just before reperfusion through 24 hours after reperfusion. At the time of sacrifice, blood was collected by orbital puncture, and the left kidney was harvested for further analysis.

At the time of reperfusion, mice were administered $100 \mu \mathrm{g}$ recombinant human IGF-1 subcutaneously $(n$ $=10), 30 \mu \mathrm{g}$ ZVAD-fmk in 1\% DMSO intraperitoneally $(n=10)$, or $20 \mu \mathrm{g}$ recombinant human EGF dissolved in $0.3 \mathrm{~mL}$ PBS subcutaneously $(n=10)$. In separate groups, $15 \mu \mathrm{g}$ LY294002 in $0.3 \mathrm{~mL}$ PBS containing 1\% DMSO was administered intraperitoneally with $(n=$ $10)$ or without $(n=10)$ IGF-1 treatment. Also in separate groups, IGF-1 $(n=10)$ or ZVAD-fmk $(n=10)$ were administered under similar conditions except after 2 hours of reperfusion. When compared with PBS treatment, neither the solvent DMSO nor treatment with 30 $\mu \mathrm{g} \mathrm{Z-Phe-Ala-Ch}{ }_{2} \mathrm{~F}$ (ZFA-fmk) in 1\% DMSO (as a negative control for the caspase-inhibiting properties of ZVAD-fmk) affected the outcome of performed experiments (data not shown). Therefore, the control group was treated subcutaneously with a vehicle consisting of $0.3 \mathrm{~mL}$ PBS $(n=12)$. A sham-operated group $(n=12)$ was subjected to the same surgical procedure without clamping the renal pedicle, and then was treated with PBS and sacrificed at corresponding time points. The dosages selected were based on pilot experiments with respect to ZVAD-fmk and LY294002 (data not shown) and on studies in which IGF-1 or EGF was found to attenuate renal reperfusion injury (6).

Assays for myeloperoxidase and blood urea nitrogen. To quantify the extent of renal neutrophil accumulation, renal myeloperoxidase (MPO) content was determined. Snap-frozen samples were thawed, homogenized for 30 seconds using a tissue homogenizer (T25B; IKA-Werke GmbH, Staufen, Germany) at 20,000 $\mathrm{g}$ in $0.5 \%$ hexadecyltrimethylammonium bromide dissolved in $50 \mathrm{mM}$ PBS ( $\mathrm{pH}$ 6.0), and then made up to concentrations of $0.17 \mathrm{~g}$ renal tissue $/ \mathrm{mL}$. After a 2-hour heat incubation in 

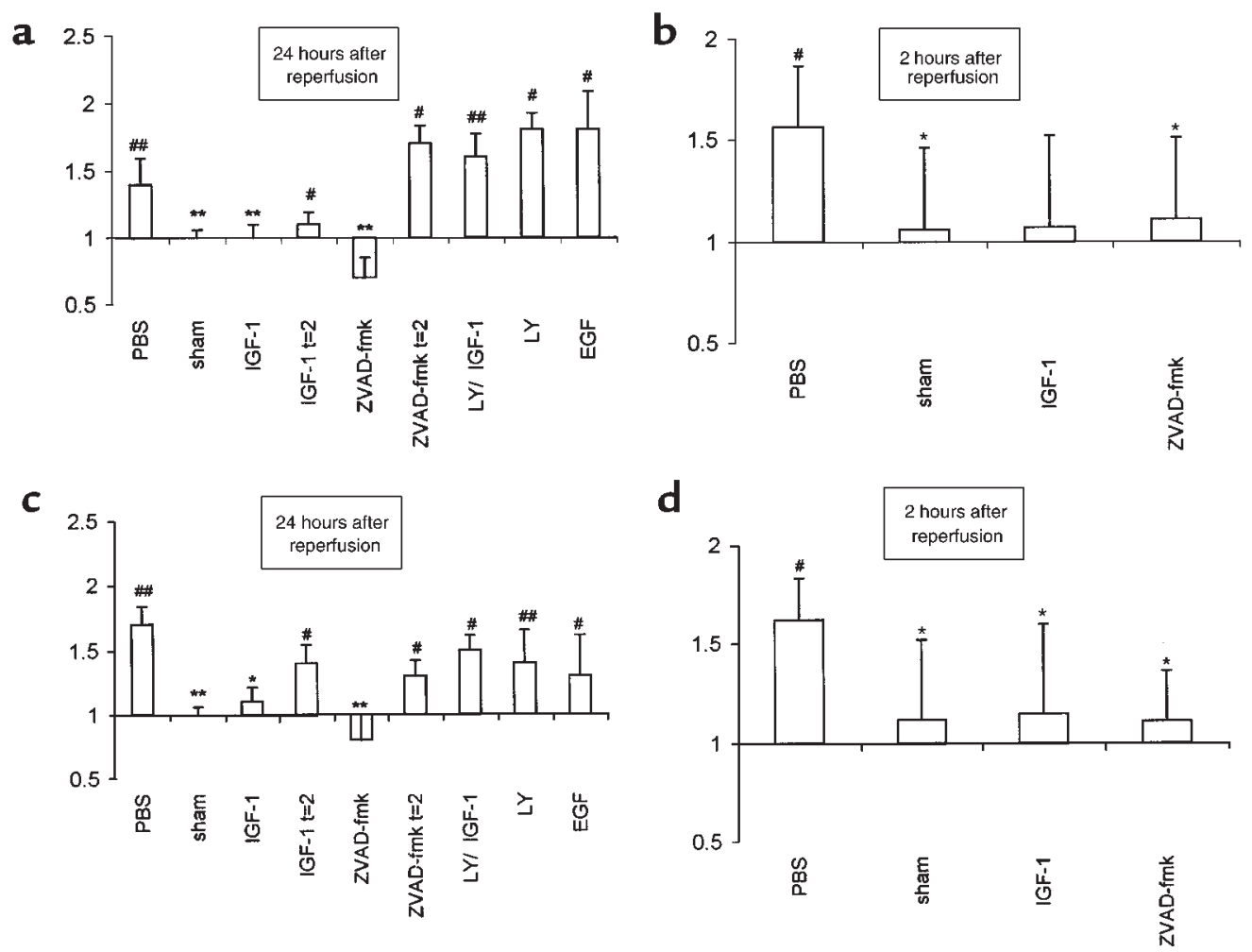

\section{Figure 2}

Renal caspase-like activities were determined kinetically in homogenates of tissue obtained after 24 hours (a and $\mathbf{c})$ and 2 hours (b and $\mathbf{d})$ of renal reperfusion in a fluorogenic substrate assay in which Ac-YVAD-amc (caspase-1-like) (a and b) or Ac-DEVD-amc (caspase-3-like) (c and $\mathbf{d}$ ) served as substrates. Note that these data may represent a more generalized form of caspase activation, as outlined in the main text. Data are expressed as the increase in fluorescence as a function of time, normalized against data obtained from the sham-operated group. The groups that indicate $t=2$ on the $x$-axis received the indicated treatment after 2 hours of reperfusion. All other groups were treated at the time of reperfusion. ${ }^{*} P<0.05,{ }^{*} P<0.01$ vs. control-treated animals. ${ }^{*} P<0.05, \# \# P<0.01$ vs. contralateral control kidney. The data shown are mean \pm SEM.

a $60^{\circ} \mathrm{C}$ water bath to verify MPO heat resistance, samples were frozen and thawed to release cellular MPO. Next, samples were spun at $11,000 \mathrm{~g}$ for 5 minutes, and supernatants were then collected. Enzymatic detection of MPO was performed in a 96-well plate (CorningCostar Corp., Cambridge, Massachusetts, USA). Assay mixtures consisted of $40 \mu \mathrm{L} \mathrm{H}_{2} \mathrm{O}_{2}$ (final concentration: $0.3 \mathrm{mM}$ ) in $80 \mathrm{mM} \mathrm{PBS}$ (pH 5.4) and $40 \mu \mathrm{L}$ in $0.5 \%$ hexadecyltrimethylammonium bromide in $50 \mathrm{mM}$ PBS $(\mathrm{pH}$ 6.0) containing diluted sample. The reaction was initiated by addition of $20 \mu \mathrm{L}$ tetramethylbenzidine (final concentration: $1.6 \mathrm{mM}$ ) in DMSO and stopped after $15 \mathrm{~min}$ utes by the addition of $50 \mu \mathrm{L} /$ well of $1 \mathrm{M} \mathrm{H}_{2} \mathrm{SO}_{4}$. Subsequently, OD was determined at $450 \mathrm{~nm}$. All samples were assayed in triplicate. MPO activity was calculated per milligram of renal tissue by comparing OD of sample wells with a titration curve of horseradish peroxidase. The obtained relative MPO activities were standardized with respect to wet/dry ratios of the assayed renal tissue. The activities are presented relative to the amount of MPO present in the contralateral kidney, which was harvested immediately after reperfusion and normalized with respect to the MPO increase at day 1 in PBS-treated mice subjected to I/R. Blood urea nitrogen (BUN) content was measured in serum obtained by orbital puncture at the time of sacrifice using a BUN Unimate 5 kit in a Cobas Fara autoanalyzer (HoffmannLa Roche Ltd., Basel, Switzerland).

Apoptosis assays. Histological aspects of apoptosis were studied by standard TdT-mediated dUTP nick end labeling (TUNEL histology). To this end, $8-\mu \mathrm{m}$ frozen sections were fixed for 30 minutes in $4 \%$ buffered formaldehyde, washed in PBS, pretreated for 10 minutes with PBS containing $0.6 \% \mathrm{H}_{2} \mathrm{O}_{2}$, and then washed again. DNA fragments were elongated and labeled with TdT and digoxigenin 11-dUTP. Subsequently, sections were treated with sheep anti-digoxigenin antibodies and washed. Finally, peroxidaselabeled rabbit anti-sheep antibodies, preincubated for 30 minutes in $10 \%$ murine serum, were applied and, after washing, developed with diaminobenzidine substrate. Sections that were pretreated with DNase 1 to nick all DNA served as positive controls.

To quantify renal caspase activity, fluorogenic substrates were used with predominant caspase-1- and caspase-3-like affinities. Samples were homogenized, snap-frozen, and then stored at $-70^{\circ} \mathrm{C}$ in a buffer containing $200 \mathrm{mM} \mathrm{NaCl}, 10 \mathrm{mM}$ Tris- $\mathrm{HCl}$ ( $\mathrm{pH}$ 7.0), $5 \mathrm{mM}$ EDTA, $10 \%$ glycerol, $1 \mathrm{mM}$ PMSF, $0.1 \mu \mathrm{M}$ aprotinin, $1.0 \mu \mathrm{M}$ leupeptin, and $5 \mathrm{mM}$ oxidized glutathione. 


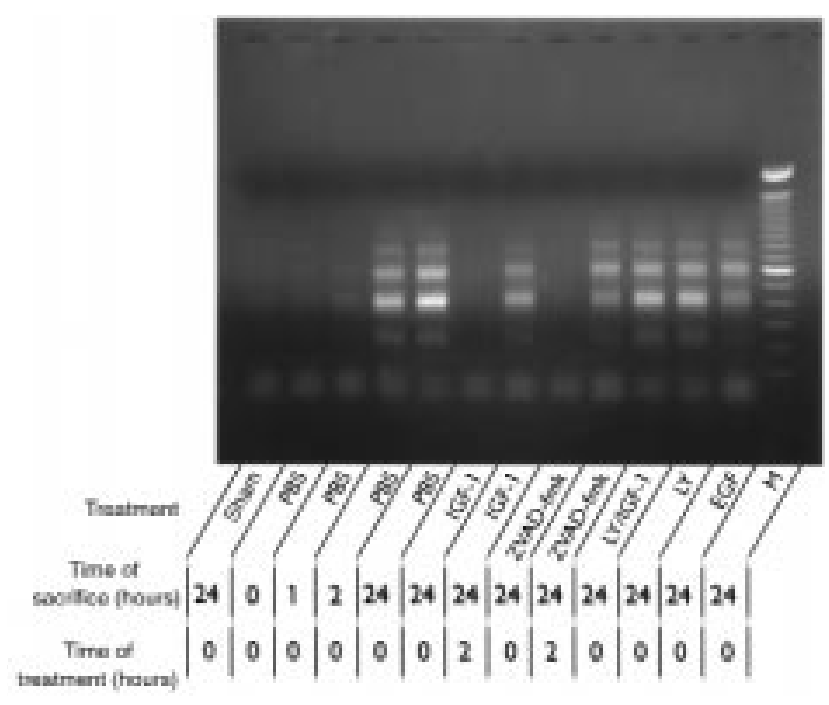

Figure 3

The extent of renal apoptosis in different treatment groups is also reflected by the presence of fragmented DNA (as a result of internucleosomal DNA cleavage), amplified by LM-PCR and then viewed on ethidium bromide-stained gel. M, molecular weight markers (range: $100-2,000$ bp).

Renal lysates (containing $40 \mu \mathrm{g}$ total protein) were incubated with $50 \mu \mathrm{M}$ of the fluorogenic substrates AcYVAD-amc (caspase-1-like) or Ac-DEVD-amc (caspase3 -like) in a cell-free system buffer containing $10 \mathrm{mM}$ HEPES (pH 7.4), $220 \mathrm{mM}$ mannitol, $68 \mathrm{mM}$ sucrose, 2 $\mathrm{mM} \mathrm{NaCl}, 2.5 \mathrm{mM} \mathrm{KH}_{2} \mathrm{PO}_{4}, 0.5 \mathrm{mM}$ EGTA, $2 \mathrm{mM}$ $\mathrm{MgCl}_{2}, 5 \mathrm{mM}$ pyruvate, $0.1 \mathrm{mM}$ PMSF, and $1 \mathrm{mM}$ DTT. The release of fluorescent 7-amino-4-methylcoumarin was measured for 1 hour at 2-minute intervals by spectrofluorometry (Cytofluor; PerSeptive Biosystems, Framingham, Massachusetts, USA). Data are expressed as the increase in fluorescence as a function of time, and then normalized against the data obtained from the sham-operated group.

The presence of internucleosomal DNA cleavage in kidneys was investigated with a commercially available ligase-mediated PCR (LM-PCR) assay kit (Apoalert; CLONTECH Laboratories Inc., Palo Alto, California, USA), enabling semiquantitative measurement of the extent of apoptosis. In brief, DNA was isolated from tissue samples (frozen previously at $-70^{\circ} \mathrm{C}$ ) using a commercially available DNA purification kit (Wizard; Promega Corp., Madison, Wisconsin, USA) according to the manufacturer's instructions. DNA purity and concentration were determined by electrophoresis through a $0.8 \%$ agarose gel containing ethidium bromide, followed by observation under ultraviolet illumination, as well as by measuring absorbance at 260/280 nm. Dephosphorylated adaptors were ligated to $5^{\prime}$ phosphorylated blunt ends with T4 DNA ligase (during 16 hours at $16^{\circ} \mathrm{C}$ ); they then served as primers in LM-PCR under the following conditions: hot start $\left(72^{\circ} \mathrm{C}\right.$ for 8 minutes, $\mathrm{Taq}$ polymerase added after $3 \mathrm{~min}-$ utes), 25 cycles $\left(94^{\circ} \mathrm{C}\right.$ for 15 seconds, and $72^{\circ} \mathrm{C}$ for 180 seconds), and postcycling $\left(72^{\circ} \mathrm{C}\right.$ for 15 minutes). To confirm that equal amounts of DNA were used for PCR, an internal control that consisted of DNA amplification with En-2 primer pairs was performed. Amplified DNA was subjected to gel electrophoresis on a 1.2\% agarose gel containing ethidium bromide.

Histology. Histological aspects of apoptosis were studied to verify that $\mathrm{I} / \mathrm{R}$ also induced morphological characteristics of apoptosis. Kidneys were fixed in situ by perfusion with $2.5 \%$ glutaraldehyde in phosphate buffer through a left ventricular cardiac catheter in anesthetized mice after 3 hours of reperfusion. After perfusion fixation, renal specimens containing outer medulla segments were fixed in $2.5 \%$ glutaraldehyde overnight. Next, segments were postfixed in $1 \%$ osmium tetroxide, dehydrated in graded alcohols, and then embedded in Epon 812 plastic. Finally, $1-\mu \mathrm{m}$ sections were stained with toluidine blue for light microscopic examination.

From animals in the different treatment groups, specimens of harvested kidneys were immediately frozen and stored at $-70^{\circ} \mathrm{C}$. Next, $5-\mu \mathrm{m}$ sections were stained for neutrophils with $\mathrm{mAb}$ Gr-1, using peroxidase-labeled goat anti-rat IgG as the secondary detection $\mathrm{mAb}$ and 3-amino-9-ethylcarbazole as a chromogen followed by a hematoxylin counterstain. To block nonspecific peroxidase activity, sections were pretreated for 10 minutes with PBS containing 0.03\% $\mathrm{H}_{2} \mathrm{O}_{2}$. No significant staining was detected in slides incubated with control $\mathrm{mAb}$ instead of the primary detecting $\mathrm{mAb}$.

Western blot analysis. Frozen samples of harvested kidneys were homogenized in a buffer containing $200 \mathrm{mM}$ $\mathrm{NaCl}, 10 \mathrm{mM}$ Tris-HCl ( $\mathrm{pH}$ 7.0), 5 mM EDTA, 10\% glycerol, $1 \mathrm{mM}$ PMSF, and $0.1 \mu \mathrm{M}$ aprotinin. After centrifugation at $15,000 \mathrm{~g}$, protein in the supernatants was quantified by the Bradford method and boiled in Laemmli buffer. Then, $35 \mu \mathrm{g}$ of protein per lane was electrophoresed in 15\% SDS-PAGE. Proteins were transferred to nitrocellulose membranes, which were subsequently blocked with $\mathrm{PBS} / 5 \%$ nonfat dry milk and then incubated for 3 hours with EMAP-II antiserum (SA 2846; diluted 1:2,000 in PBS/0.1\% Tween20). After washing, the membranes were incubated for 2 hours with peroxidase-labeled goat anti-rabbit IgG (diluted 1:2,000 in PBS/0.1\% Tween 20), washed, and then developed by enhanced chemiluminescence.

Statistics. Data are expressed as the mean \pm SEM, and statistical analysis was performed by Student's $t$ test. $P$ values less than 0.05 were considered statistically significant.

\section{Results}

Renal I/R induces apoptosis and subsequent inflammation. To determine the extent of apoptosis, and to exclude necrosis or mechanisms independent from apoptosis as main contributors to DNA fragmentation, different techniques were used. Kidneys were investigated by TUNEL histology to localize DNA fragmentation after 
reperfusion. For morphological assessment of apoptosis, toluidine blue histology was performed using plastic-embedded tissues. Also, caspase-like activities and internucleosomal DNA cleavage were measured in homogenates of kidneys obtained after reperfusion.

TUNEL-positive nuclei, restricted mainly to the tubular epithelial cells of the outer medulla, were identified in biopsies obtained after as little as 2 hours of reperfusion (Figure 1, $a$ and $b$ ). At this time point, TUNEL histology revealed the extracellular presence of apoptotic bodies, which were often extruded into the tubular lumen (Figure 1c). In a separate group of mice, sacrificed after 3 hours of reperfusion, toluidine blue-stained $1-\mu \mathrm{m}$ sections showed dense condensation of nuclear chromatin and nuclear fragmentation in apoptotic tubular epithelial cells (Figure 1, $\mathrm{d}$ and e). In addition, increased renal caspase-1- and caspase3-like activities (Figure 2, b and d), as well as apparent internucleosomal DNA cleavage (Figure 3), were observed after 2 hours of reperfusion.

As documented previously (4), after 1 day of reperfusion the number of tubular epithelial cells containing TUNEL-positive nuclei had increased, and TUNELpositive nuclei of infiltrating immune cells were observed (data not shown). Also at this time, increased renal caspase-1- (Figure 2a) and caspase-3-like (Figure $2 c)$ activities were detected. Together with the observed occurrence of internucleosomal DNA cleavage (Figure 3), these findings suggest the presence of ongoing renal apoptosis starting as early as 2 hours after reperfusion.

IGF-1 abrogates early apoptosis, impaired renal function, and $I / R$-induced inflammation. We first investigated the relation between I/R-induced apoptosis and inflammation by using IGF-1, because pilot experiments revealed that this agent prevented I/R-induced apoptosis in murine kidneys. Administration of IGF-1 at the time of reperfusion - as opposed to administration of PBS - prevented apoptosis, as indicated by a decreased number of TUNEL-positive nuclei (data not shown), decreased caspase-like activities (Figure 2), and the absence of apparent internucleosomal DNA cleavage (Figure 3). These data reveal the antiapoptotic potential of IGF-1 in an early phase after renal ischemia.

Next, we studied whether abrogation of early apoptosis affects the later consequences of renal I/R. IGF-1 administration at the time of reperfusion prevented the $\mathrm{I} / \mathrm{R}$-induced impairment of renal function, as reflected by increased BUN content at day 1 (Figure 4). Moreover, IGF-1 treatment limited I/R-induced inflammation at that time point by reducing the renal influx of neutrophils, as reflected by the presence of Gr-1-positive cells (Figure 5, a and b) and the increased renal MPO activity (Figure $5 \mathrm{c}$ ). Taken together, these findings indicate that exogenous IGF-1 prevents I/R-induced apoptosis, impairment of renal function, and inflammation.

Protection from I/R-induced apoptosis by IGF-1 is mediated by phosphatidylinositol 3-kinase. Phosphatidylinositol 3kinase (PI 3-kinase), an important component of signal transduction by insulin family receptors, has been

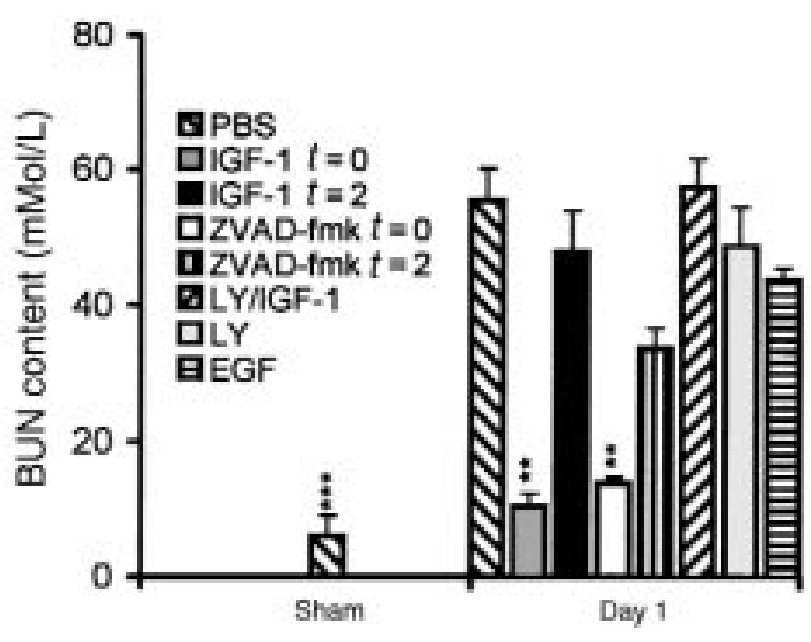

Figure 4

Renal function in the different experimental groups as reflected by BUN content. ${ }^{*} P<0.01,{ }^{*} * P<0.001$ vs. control-treated animals. The data shown are mean \pm SEM.

reported to mediate antiapoptotic signaling by IGF-1 in vitro (7). To investigate more closely the mechanisms by which IGF-1 attenuates reperfusion-induced apoptosis and inflammation, we treated mice with a combination of IGF-1 and LY294002 (a selective PI 3-kinase inhibitor) (8). In contrast to IGF-1 treatment, combined administration of IGF-1 and LY294002 at the time of reperfusion failed to prevent apoptosis. Animals treated with this combination exhibited an abrogation of IGF-1-induced inhibition of apoptosis, resulting in increased numbers of TUNEL-positive nuclei (data not shown), significant caspase-like activity (Figure 2), as well as apparent internucleosomal DNA cleavage (Figure 3 ) at day 1 after I/R. Moreover, at this time point, they exhibited decreased renal function (Figure 4) and an increased renal neutrophil influx (Figure 5c) when compared with IGF-1-treated animals. LY294002 treatment, compared with PBS treatment, did not result in significant differences in any of the parameters evaluated (Figures 2-5). These data demonstrate that in vivo antiapoptotic signaling by IGF-1 is mediated by the PI 3-kinase pathway.

Inflammation is dependent on antecedent apoptosis after renal $I / R$. Besides antiapoptotic properties, IGF-1 has a well-documented mitogenic potential that may contribute to the repair and recovery of ischemically injured renal tissue. To address the question whether the mitogenic effects of IGF-1 were responsible for the observed prevention of apoptosis and inflammation in our model, mice were treated with EGF, a powerful mitogen for renal epithelial cells. EGF administered at the time of reperfusion failed to prevent reperfusioninduced renal apoptosis, as reflected by increased numbers of TUNEL-positive nuclei (data not shown), significant caspase-like activity (Figure 2), and apparent internucleosomal DNA cleavage (Figure 3) at day 1. Hence, the absence of any antiapoptotic effect excludes the possibility that EGF, as opposed to IGF-1, func- 

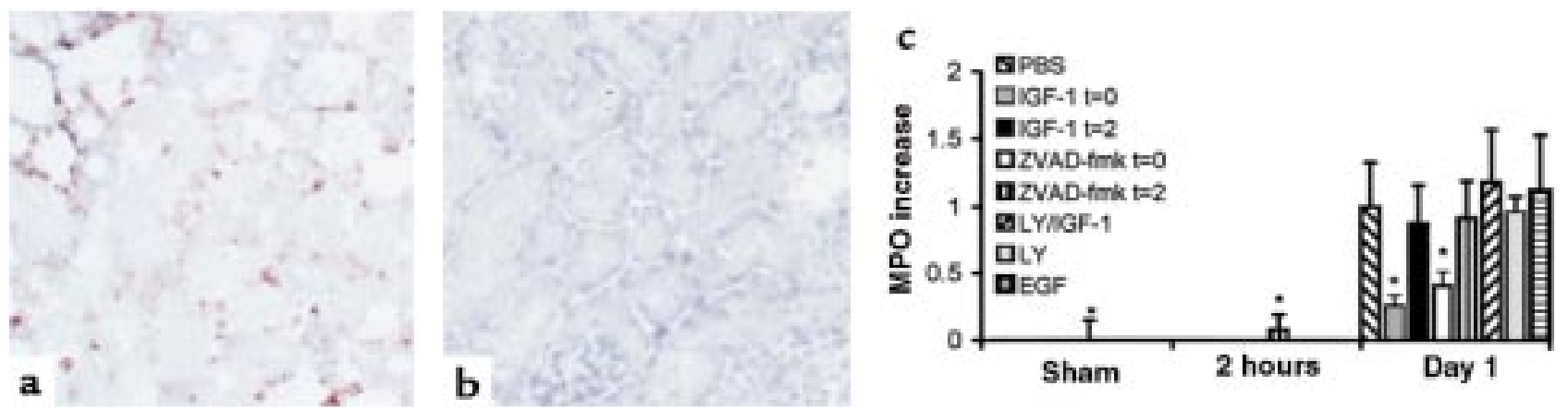

Figure 5

Representative light micrographs ( $\mathbf{a}$ and $\mathbf{b}$ ) showing infiltrating neutrophils in areas with impaired renal morphological integrity in biopsies obtained from mice subjected to renal I/R after 1 day of reperfusion (a). Neutrophils were stained with the anti-murine neutrophil mAb Gr-1. Neither loss of morphological integrity nor infiltrating neutrophils were observed in kidneys obtained from sham-operated mice (b) (×200). Neutrophil influx was assessed quantitatively by determination of MPO increase at 2 hours and 1 day of reperfusion (c). Values are presented relative to the amount of MPO present in the contralateral kidney harvested immediately after reperfusion, and then normalized with respect to the MPO increase at day 1 in PBS-treated mice subjected to I/R. ${ }^{*} P<0.05 \mathrm{vs}$. control-treated animals. The data shown are mean \pm SEM.

tions as a survival factor in the model used. Moreover, as opposed to IGF-1, EGF did not prevent I/R-induced impaired renal function (Figure 4) or inflammation (Figure 5c) at day 1.

To investigate whether the anti-inflammatory effect of exogenous IGF-1 is a direct effect rather than a consequence of the absence of apoptosis, mice were treated with the tripeptide caspase inhibitor ZVAD-fmk at the time of reperfusion, as well as after 2 hours of reperfusion. At the later time point, the presence of renal apoptosis is evident (Figure 1), whereas an inflammatory response, as defined by the present parameters, cannot yet be distinguished (Figure $5 \mathrm{c}$ ). In line with a recent report on cardiac I/R (9), we show that ZVAD-fmk administered at the time of reperfusion completely abrogated renal apoptosis (Figures 2 and 3). In addition, ZVAD-fmk administered at the time of reperfusion prevented the impairment of renal function (Figure 4) and the development of the inflammatory response (Figure $5 c)$ at day 1 . In contrast, ZVAD-fmk administered after 2 hours of reperfusion not only failed to attenuate the extent of the already initiated apoptotic response (Figures 2 and 3), but it also failed to attenuate subsequent impairment of renal function (Figure 4) and the inflammatory response (Figure 5c). Similarly, at day 1, no reversal of renal apoptosis could be detected in mice that received IGF-1 after 2 hours of reperfusion (Figures 2 and 3); in addition, these animals exhibited signs of inflammation (Figure 5c) and impaired renal function (Figure 4), as seen in PBS-treated animals. These findings indicate that development of inflammation is dependent on antecedent apoptosis after I/R. Since abrogation of apoptosis by IGF-1 or ZVAD-fmk limits $\mathrm{I} / \mathrm{R}$-induced inflammation, apoptosis is a likely candidate for induction of inflammation in the model used.

Posttranslational processing of EMAP-II coincides with I/Rinduced apoptosis and is inhibited by IGF-1 and ZVAD-fmk. The precursor form of EMAP-II can serve as a target substrate for activated caspases (10). We used Western blotting to investigate the effect of $I / R$ on posttranslational processing of EMAP-II. Figure 6 shows that I/R results in enhanced cleavage of the 43-kDa EMAP-II precursor as early as 2 hours of reperfusion. The active 23-kDa cleavage product remained detectable in kidneys subjected to 24 hours of reperfusion. Hence, the formation of functional EMAP-II coincides with the manifestation of apoptosis after renal I/R. EMAP-II activation was clearly inhibited in kidneys obtained from sham-operated animals or from mice treated with ZVAD-fmk at the time of reperfusion, after 2 hours (data not shown) or 24 hours (Figure 6) of reperfusion. At these time points, animals that were treated with IGF-1 at the time of reperfusion exhibited only lowintensity 23-kDa bands (Figure 6). Digital image analysis of activated EMAP-II protein band intensities of the representative EMAP-II Western blot (Figure 6) reveals that the intensity of the $23-\mathrm{kDa}$ band after IGF- 1 treatment is approximately $5 \%$ of the $23-\mathrm{kDa}$ band intensity 24 hours after PBS treatment. These findings are in line with the other parameters measured in IGF1-treated animals. As shown in Figure 6, the formation of $23-\mathrm{kDa}$ EMAP-II also resulted in formation of bands representing a molecular mass between 23 and $43 \mathrm{kDa}$, which has been suggested to be the result of intermediate cleavage product formation (10). These results demonstrate that renal I/R induces EMAP-II activation. Both IGF-1 and the caspase inhibitor ZVAD-fmk strongly reduce EMAP-II activation, which may explain the observed ability of these agents to inhibit the apoptosis-induced inflammatory response after renal I/R.

\section{Discussion}

We hypothesized that apoptosis after I/R constitutes a potential trigger of inflammation, and we studied this possibility by using inhibitors of the apoptotic response in a murine model of renal I/R. Evidence for the early involvement of apoptosis is provided by the presence of DNA fragmentation, morphological features character- 
istic for apoptosis, as well as increased caspase-1- and caspase-3-like activities in kidneys after 2 hours of reperfusion. These findings are in line with various in vivo and in vitro reports showing that renal apoptosis after ischemia is induced by hypoxia (11) and ATP depletion (12). In addition, hydrolyzation of the plasma membrane component sphingosine, and consequent liberation of ceramide, has been reported to initiate apoptosis after as little as 30 minutes of renal reperfusion (13). Furthermore, during I/R-induced inflammation at day 1, upregulated Fas (capable of inducing apoptosis independent of ceramide signaling) (4), TNF- $\alpha$ (14), and P53 (15) have been associated with I/R-induced apoptosis. In contrast, our in vivo findings clearly differ from data presented by Ueda et al. (16), who reported that anoxia followed by reoxygenation of isolated rat proximal tubules in vitro may occur without morphological aspects of apoptosis.

The present findings show that mice treated with a single dose of IGF-1 at the time of reperfusion exhibited abrogation of apoptosis, as demonstrated by decreased numbers of TUNEL-positive nuclei, decreased renal caspase-like activities, and the absence of apparent internucleosomal DNA cleavage. These results confirm that IGF-1 is a potent inhibitor of reperfusion-induced apoptosis. In addition, ZVAD-fmk, a broad spectrum inhibitor of activated caspases, abrogated I/R-induced apoptosis, whereas ZFA-fmk, a homologous peptide lacking the caspase-inhibiting properties of ZVAD-fmk, failed to prevent apoptosis. Hence, inhibition of apoptosis prevented the loss of kidney function and the development of an inflammatory response. In contrast, administration of antiapoptotic IGF-1 or ZVAD-fmk after 2 hours of reperfusion (after the onset of apoptosis) failed to prevent loss of kidney function and inflammation. Few studies have addressed the potential of IGF-1 in ischemia-induced apoptosis $(17,18)$. The latter report $(18)$ assumed a distinct "antineutrophil property" apart from the antiapoptotic effects of IGF-1. In contrast, our findings clearly define apoptosis as an event arising proximal to the onset of $\mathrm{I} / \mathrm{R}$-induced inflammation.

We investigated whether mechanisms other than the inhibition of apoptosis contributed to the anti-inflammatory and renal function-improving effects of IGF-1 and ZVAD-fmk. Caspase-1- and caspase-3-like activities during I/R-induced apoptosis were significantly prevented by IGF-1 or ZVAD-fmk administered at the time of reperfusion. Whereas caspase-3 activation reflects many forms of apoptosis (19), a functional role for caspase-1 during apoptosis appears to be restricted to cell death induced by specific agents such as FasL (20) and granzyme B (21). Caspase-1, also referred to as IL-1-converting enzyme (ICE), is involved in, but not essential to, the maturation of IL-1 and IL-18 $(22,23)$. Therefore, activated caspase-1-induced release of IL-1 and/or IL-18 may contribute to the inflammatory response after I/R (23). However, inhibition of endogenous IL-1 (ref. 24 and our own unpublished observations) and IL-18 (25) only minimally decreased renal influx of neutrophils and loss

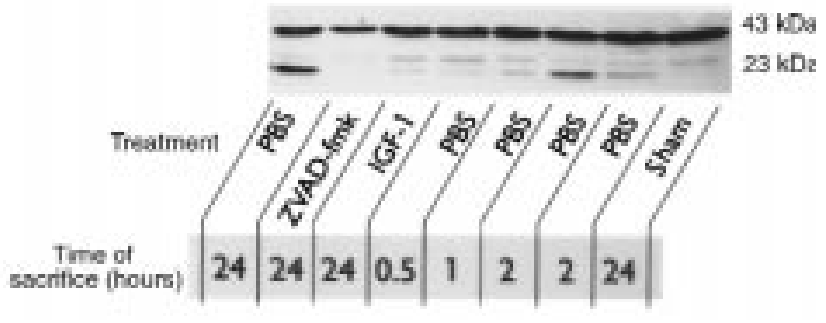

\section{Figure 6}

Posttranslational processing of the EMAP-II protein is induced in parallel with apoptosis after as little as 2 hours of reperfusion. Western blots were performed with protein isolated from kidneys in mice that were sacrificed after different periods of reperfusion. Incubation with the EMAP-II antiserum SA 2846 revealed constitutive expression of inactive pro-EMAP-II, which resulted in a 43-kDa band. After 2 hours of reperfusion, the presence of $23-\mathrm{kDa}$ mature EMAP-II was observed, which was even more apparent after 24 hours of reperfusion. Antiapoptotic treatment at the time of reperfusion with IGF-1 or ZVAD-fmk evidently inhibited I/R-induced EMAP-II maturation.

of kidney function after 1 day of reperfusion when compared with administration of IGF-1 or ZVAD-fmk (data not shown). Finally, it has to be taken into account that the measured renal caspase-like activities are likely to represent a more generalized form of caspase activation, because enzymatic activities of caspase- 1 and caspase- 3 on Ac-YVAD-amc and Ac-DEVD-amc have been shown to be not fully specific (26).

The anabolic effect attributed to IGF-1, putatively leading to enhanced tissue repair, may add to the therapeutic effect of IGF-1 (6). Moreover, IGF-1-induced renal nitric oxide (NO) can improve kidney function by directly enhancing renal circulation (27), as well as by contributing to limitation of apoptosis through inhibition of caspases (28). However, direct anti-inflammatory effects of IGF-1 have not been reported previously. The present findings show that neither treatment with EGF, a renotropic growth factor with anabolic effects similar to IGF-1 (29), nor treatment with IGF-1 or ZVAD-fmk after 2 hours of reperfusion attenuated the loss of kidney function or inflammation.

Similar to in vitro observations $(7,30)$, we further showed, by coadministrating the PI 3-kinase inhibitor LY294002, that PI 3-kinase constitutes an essential component of IGF-1 receptor-mediated, antiapoptotic signaling in vivo. Taken together, these results demonstrate that the anti-inflammatory effects of IGF-1 and ZVAD-fmk are distinctively mediated by antiapoptotic signaling rather than by inhibition of IL-1 and/or IL-18 maturation, anabolic stimulation, or NO-induced enhanced kidney function. Nevertheless, despite the lack of an apparent functional role for activated caspase- 1 in our model, our findings do not definitely rule out the possibility that apoptosis and inflammation are 2 largely independent processes that share thus far unknown upstream molecular mechanisms.

The present findings demonstrate that inhibition of apoptosis after renal I/R prevents subsequent inflammation. This apparently shows apoptosis to be critical 
for the induction of inflammation in the present model. Our results also demonstrate that $\mathrm{I} / \mathrm{R}$ induces activation of EMAP-II. Inactive pro-EMAP-II may serve as a target substrate for activated caspases (10), which we presently show to be upregulated as a consequence of I/R. The evident formation of mature EMAP-II coinciding with elaboration of I/R-induced apoptosis, as well as the observed decrease in EMAP-II activation after IGF-1 or ZVAD-fmk treatment, is in line with a recent report of Knies et al. (10). This study showed that the release of mature EMAP-II is directly linked to apoptosis and can be abrogated in vitro by caspase inhibition. Mature EMAP-II is a potent chemokine that stimulates migration of polymorphonuclear neutrophils and monocytes in vivo, release of tissue factor on endothelial cells (31), and TNF- $\alpha$ from monocytes (32). Its peptide sequence exhibits strong regional homology with other proinflammatory mediators such as human von Willebrand factor antigen II (33) and IL-8 (34). Furthermore, it was recently demonstrated that mediators of apoptosis can cleave aminoacyl-tRNA synthetase, which subsequently leads to release of proinflammatory cleavage products similar to EMAP-II, like the $\mathrm{NH}_{2}$-terminal catalytic domain of tyrosyl tRNA synthetase (35).

The present data suggest that EMAP-II maturation, as a consequence of $\mathrm{I} / \mathrm{R}$-induced apoptosis, contributes to recruitment of inflammatory cells to the reperfused kidney. However, more studies are needed to determine the functional role of EMAP-II in I/R and to elucidate other mechanisms by which apoptosis induces an inflammatory response. The absence of sufficient phagocytosis of apoptotic cells has been associated with further degeneration, and ultimately necrosis, of apoptotic cells, a process termed secondary necrosis $(36,37)$. This secondary necrosis has been suggested to occur when the extent of necrosis overwhelms the phagocytic capacity of the tissue (38), and it appears to be another important link between apoptosis and inflammation. It is tempting to conclude that phagocytosis cannot deal sufficiently with sudden widespread apoptosis within an organ subjected to prolonged ischemia, which ultimately leads to secondary necrosis and inflammation.

Many documented attempts to directly reduce the I/Rinduced inflammatory response in various models have unmasked inflammation as a significant cause of subsequent tissue injury and dysfunction $(1,14)$. The present studies indicate that early apoptosis is a crucial event in the process that initiates inflammation and subsequent tissue injury. This is because abrogation of early I/Rinduced apoptosis prevents the development of inflammation and organ dysfunction. This concept of apoptosis-induced inflammation after I/R puts forth new, important opportunities to effectively prevent clinical manifestations of reperfusion injury. Such therapies may include, prevention of complications arising from the use of ischemically damaged donor organs, cardiopulmonary bypass surgery, aortic cross-clamping, and circulatory shock. Agents such as IGF-1 (which can be safely admin- istered to humans) and ZVAD-fmk, as well as new antiapoptotic substances currently under investigation, may provide new therapeutic means to treat these conditions.

\section{Acknowledgments}

We thank F. le Noble (Department of Physiology, University of Maastricht) and P. Frederik and P. Bomans (both from Electron Microscopy Unit, University of Maastricht) for expert technical assistance. This study was supported by grant C98.1719 from the Dutch Kidney Foundation, and by grant PL962107 from the European Community Biotechnology program.

1. Vedder, N.B., et al. 1990. Inhibition of leukocyte adherence by anti-CD18 monoclonal antibody attenuates reperfusion injury in the rabbit ear. Proc. Natl. Acad. Sci. USA. 87:2643-2646.

2. Shreeniwas, R., et al. 1992. Hypoxia-mediated induction of endothelial cell interleukin-1 alpha. An autocrine mechanism promoting expression of leukocyte adhesion molecules on the vessel surface. J. Clin. Invest. 90:2333-2339.

3. Metinko, A.P., Kunkel, S.L., Standiford, T.J., and Strieter, R.M. 1992. Anoxia-hyperoxia induces monocyte-derived interleukin-8. J. Clin. Invest. 90:791-798

4. Nogae, S., et al. 1998. Induction of apoptosis in ischemia-reperfusion model of mouse kidney: possible involvement of Fas. J. Am. Soc. Nephrol. 9:620-631.

5. Fadok, V.A., et al. 1998. Macrophages that have ingested apoptotic cells in vitro inhibit proinflammatory cytokine production through autocrine/paracrine mechanisms involving TGF-beta, PGE2, and PAF. J. Clin. Invest. 101:890-898

6. Goes, N., Urmson, J., Vincent, D., Ramassar, V., and Halloran, P.F. 1996. Effect of recombinant human insulin-like growth factor-1 on the inflammatory response to acute renal injury. J. Am. Soc. Nephrol. 7:710-720.

7. Kulik, G., Klippel, A., and Weber, M.J. 1997. Antiapoptotic signalling by the insulin-like growth factor I receptor, phosphatidylinositol 3-kinase, and Akt. Mol. Cell. Biol. 17:1595-1606.

8. Vlahos, C.J., Matter, W.F., Hui, K.Y., and Brown, R.F. 1994. A specific inhibitor of phosphatidylinositol 3-kinase, 2-(4-morpholinyl)-8-phenyl4H-1-benzopyran-4-one (LY294002). J. Biol. Chem. 269:5241-5248.

9. Yaoita, H., Ogawa, K., Maehara, K., and Maruyama, Y. 1998. Attenuation of ischemia/reperfusion injury in rats by a caspase inhibitor. Circulation. 97:276-281.

10. Knies, U.E., et al. 1998. Regulation of endothelial monocyte-activating polypeptide II release by apoptosis. Proc. Natl. Acad. Sci. USA. 95:12322-12327.

11. Beeri, R., et al. 1995. Rapid DNA fragmentation from hypoxia along the thick ascending limb of rat kidneys. Kidney Int. 47:1806-1810.

12. Lieberthal, W., Menza, S.A., and Levine, J.S. 1998. Graded ATP depletion can cause necrosis or apoptosis of cultured mouse proximal tubular cells. Am. J. Physiol. 43:F315-F327.

13. Zager, R.A., Iwata, M., Conrad, D.S., Burkhart, K.M., and Igarashi, Y. 1997. Altered ceramide and sphingosine expression during the induction phase of ischemic acute renal failure. Kidney Int. 52:60-70.

14. Daemen, M.A.R.C., Van de Ven, W.C.M., Heineman, E., and Buurman, W.A. 1999. Pro- and anti-inflammatory mechanisms in renal reperfusion injury in mice. Modulation by endogenous tumor necrosis factor alpha and interleukin-10. Transplantation. 67:792-800.

15. Raafat, A.M., et al. 1997. Calcium blockade reduces renal apoptosis during ischemia reperfusion. Shock. 8:186-192.

16. Ueda, N., Walker, P.D., Hsu, S.M., and Shah, S.V. 1995. Activation of a 15 -kDa endonuclease in hypoxia/reoxygenation injury without morphologic features of apoptosis. Proc. Natl. Acad. Sci. USA. 92:7202-7206.

17. Hirschberg, R., and Ding, H. 1998. Mechanisms of insulin-like growth factor-I-induced accelerated recovery in experimental ischemic acute renal failure. Miner. Electrolyte Metab. 24:211-219.

18. Buerke, M., et al. 1995. Cardioprotective effect of insulin-like growth factor I in myocardial ischemia followed by reperfusion. Proc. Natl. Acad. Sci. USA. 92:8031-8035.

19. Lazebnik, Y.A., Kaufmann, S.H., Desnoyers, S., Poirier, G.G., and Earnshaw, W.C. 1994. Cleavage of poly(ADP-ribose) polymerase by a proteinase with properties like ICE. Nature. 371:346-347.

20. Los, M., et al. 1995. Requirement of an ICE/CED-3 protease for Fas/APO-1-mediated apoptosis. Nature. 375:81-83.

21. Shi, L., et al. 1996. Activation of an interleukin 1 converting enzymedependent apoptosis pathway by granzyme B. Proc. Natl. Acad. Sci. USA. 93:11002-11007.

22. Ghayur, T., et al. 1997. Caspase-1 processes IFN-gamma-inducing factor and regulates LPS-induced IFN-gamma production. Nature. 386:619-623. 
23. Miwa, K., et al. 1998. Caspase 1-independent IL-1 beta release and inflammation induced by the apoptosis inducer Fas ligand. Nat. Med. 4:1287-1292.

24. Haq, M., Norman, J., Saba, S.R., Ramirez, G., and Rabb, H. 1998. Role of IL-1 in renal ischemic reperfusion injury. J. Am. Soc. Nephrol. 9:614-619.

25. Daemen, M.A.R.C., Van 't Veer, C., Wolfs, T.G.A.M., and Buurman, W.A. 1999. Ischemia-reperfusion induced IFN-gamma upregulation: involvement of IL-12 and IL-18. J. Immunol. 162:5506-5510.

26. Van de Craen, M., Declercq, W., Van den Brande, I., Fiers, W., and Vandenabeele, P. 1999. The proteolytic procaspase activation network: an in vitro analysis. Cell Death Differ. In press.

27. Noguchi, S., et al. 1993. Insulin-like growth factor-I ameliorates tran sient ischemia-induced acute renal failure in rats. J. Pharmacol. Exp. Ther. 267:919-926.

28. Li, J., Billiar, T.R., Talanian, R.V., and Kim, Y.M. 1997. Nitric oxide reversibly inhibits seven members of the caspase family via S-nitrosylation. Biochem. Biophys. Res. Commun. 240:419-424.

29. Norman, J., et al. 1987. EGF-induced mitogenesis in proximal tubular cells: potentiation by angiotensin II. Am. J. Physiol. 253:F299-F309.

30. Parrizas, M., Saltiel, A.R., and LeRoith, D. 1997. Insulin-like growth factor 1 inhibits apoptosis using the phosphatidylinositol 3'-kinase and mitogen-activated protein kinase pathways. J. Biol. Chem. 272:154-161.

31. Kao, J., et al. 1994. A peptide derived from the amino terminus of endothelial-monocyte-activating polypeptide II modulates mononuclear and polymorphonuclear leukocyte functions, defines an apparently novel cellular interaction site, and induces an acute inflammatory response. J. Biol. Chem. 269:9774-9782.

32. Kao, J., et al. 1994. Characterization of a novel tumor-derived cytokine. Endothelial-monocyte activating polypeptide II. J. Biol. Chem. 269:25106-25119.

33. Fay, P.J., et al. 1986. Propolypeptide of von Willebrand factor circulates in blood and is identical to von Willebrand antigen II. Science. 232:995-998.

34. Clark, L.I., Schumacher, C., Baggiolini, M., and Moser, B. 1991. Structure-activity relationships of interleukin-8 determined using chemically synthesized analogs. Critical role of $\mathrm{NH} 2$-terminal residues and evidence for uncoupling of neutrophil chemotaxis, exocytosis, and receptor binding activities. J. Biol. Chem. 266:23128-23134.

35. Wkasugi, K., and Schimmel, P. 1999. Two distinct cytokines released from a human aminoacyl-tRNA synthetase. Science. 284:147-150.

36. Lieberthal, W., Triaca, V., and Levine, J. 1996. Mechanisms of death induced by cisplatin in proximal tubular epithelial cells: apoptosis vs. necrosis. Am. J. Physiol. 270:F700-F708.

37. Lieberthal, W., and Levine, J.S. 1996. Mechanisms of apoptosis and its potential role in renal tubular epithelial cell injury. Am. J. Physiol. 271:F477-F488.

38. Schwartzman, R.A., and Cidlowski, J.A. 1993. Apoptosis: the biochemistry and molecular biology of programmed cell death. Endocr. Rev. 14:133-151 